\title{
Case for an Improved Effective-Atomic-Number for the Electronic Baggage Scanning Program
}

\author{
Jerel A. Smith \\ Jeffrey S. Kallman \\ Harry E. Martz Jr. \\ Lawrence Livermore National Laboratory \\ Livermore, CA 94551 \\ Work performed for the \\ Science \& Technology Directorate of the \\ Department of Homeland Security \\ Statement of Work \\ IAA HSHQPM-10-X-00045
}

October 16, 2012

LLNL-TR-520312-REV-1 ${ }^{1}$
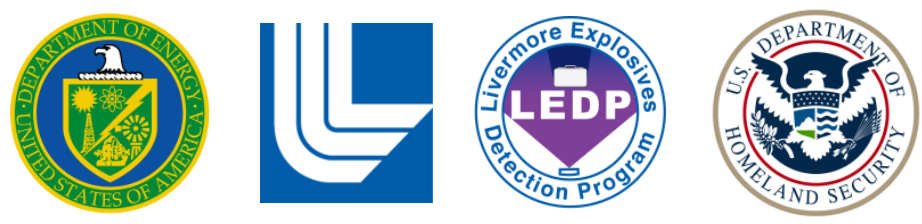

${ }^{1}$ This document is updated from LLNL-TR-520312 with the inclusion of Reference 7, minor corrections in the calculations of Ze for several materials, and the improvement of non-substantive typographical errors and wording. 
This document was prepared as an account of work sponsored by an agency of the United States government. Neither the United States government nor Lawrence Livermore National Security, LLC, nor any of their employees makes any warranty, expressed or implied, or assumes any legal liability or responsibility for the accuracy, completeness, or usefulness of any information, apparatus, product, or process disclosed, or represents that its use would not infringe privately owned rights. Reference herein to any specific commercial product, process, or service by trade name, trademark, manufacturer, or otherwise does not necessarily constitute or imply its endorsement, recommendation, or favoring by the United States government or Lawrence Livermore National Security, LLC. The views and opinions of authors expressed herein do not necessarily state or reflect those of the United States government or Lawrence Livermore National Security, LLC, and shall not be used for advertising or product endorsement purposes.

This work performed under the auspices of the U.S. Department of Energy by Lawrence Livermore National Laboratory under Contract DE-AC52-07NA27344. 


\title{
Case for an Improved Effective-Atomic-Number for the Electronic Baggage Scanning Program
}

\author{
Jerel A. Smith \\ Jeffrey S. Kallman \\ Harry E. Martz Jr. \\ Lawrence Livermore National Laboratory \\ Livermore, CA 94551
}

\subsection{Executive Summary}

$\mathrm{Z}_{\mathrm{eff}}$, a parameter representing an "effective atomic number" for a material, plays an important role in the Electronic Baggage Scanning Program (EBSP) to detect threats in dual-energy computed tomography (CT) baggage-scanning systems. We believe that Zeff, as defined and used on this program, does not provide the most accurate representation of a material's X-ray properties..

We present the case for a new method that defines an effective atomic number for compounds and mixtures, which we refer to as $\mathrm{Z}_{\mathrm{e}}$. Unlike $\mathrm{Z}_{\mathrm{eff}}, \mathrm{Z}_{\mathrm{e}}$ is tied by definition to the $\mathrm{x}$-ray absorption properties of each specific material. Use of this alternative will provide a more accurate scale for calibrating LLNL-designed Micro-CT systems and vendor-built Explosives Detection Systems (EDS) against standard reference materials, and it will provide a more accurate physical characterization of the $\mathrm{x}$-ray properties of materials evaluated on those systems.

This document,

- Describes the current usage of the $\mathrm{Z}_{\mathrm{eff}}$ parameter

- Details problems entailed in the use of the $Z_{\text {eff }}$ parameter

- Proposes a well-defined alternative- $\mathrm{Z}_{\mathrm{e}}$

- Proposes and demonstrates an algorithm for optimally associating $\mathrm{Z}_{\mathrm{e}}$ with any specified compound or mixture

- Discusses issues that can impact the usefulness of an effective- $Z$ model

- Recommends that, in order that the chosen effective-Z parameter not materially impact the accuracy of data produced by the EBSP program, the use of $Z_{\text {eff }}$ be replaced by $Z_{e}$ 


\subsection{Background}

A key objective of the LLNL component of the EBS program is to provide information on materials, particularly home-made explosives (HMEs), that EDS vendors can use to determine how each specific material would appear on their x-ray CT systems. X-ray CT systems measure an "apparent" linear-attenuation coefficient (LAC) for a material. In addition to the inherent $x$ ray properties of a sample (material density and atomic composition), the LAC depends on the thickness of the sample, the spectrum used to measure the sample absorption, and other physical characteristics of the measurement system.

The LAC is defined by the classical Beer-Lambert law relating the attenuated intensity of a source, I, to the initial intensity, $\mathrm{I}_{0}$, a path-length, $\mathrm{x}$, and the linear attenuation coefficient within that medium:

$$
I=I o \operatorname{Exp}[-\mu * x]
$$

Equation 1a

where the LAC is represented by the symbol $\mu$.

As we discuss the use of the LAC's in extracting information about HME specimens, it is helpful to keep in mind, that Equation 1a is really shorthand for a more complex expression. The hidden pieces are shown in Equation 1b.

$$
I(E x)=I o(E x) \operatorname{Exp}\left[-\frac{\mu}{\rho}(Z, E x)\right] \int \rho(x) d x
$$

Equation $1 b$

We can see that the unattenuated intensity, $\mathrm{I}_{0}$, and the attenuation coefficient are explicitly dependent on $\mathrm{x}$-ray energy, Ex. The incoming spectrum contained in $\mathrm{I}_{0}$ is a continuum, not a single energy. The transmitted spectrum present in $\mathrm{I}(\mathrm{Ex})$ is not only reduced, but changed by the amount of material encountered as shown by the integral of the material density, $\rho$, along the path, $\mathrm{x}$.

Note that the LAC, $\mu$, can be equivalently expressed in terms of $\mathrm{x}$-ray cross sections. This latter formulation is more appropriate to our approach, and will be used below to implement the proposed definition of the effective atomic number.

$$
\frac{\mu}{\rho}(Z, E x) \rho=\mu=\sigma_{e}(Z, E x) \rho_{e}
$$

Equation 1c

The mass-absorption coefficients, $\mu / \rho(Z, E x)$, and the atomic cross sections, $\sigma(Z, E x)$, have been systematically tabulated in $\mathrm{x}$-ray absorption tables [1-3]. In this document, we express the crosssections per-electron (or per moles of electron to make the numbers manageable), and refer specifically to total or "narrow-angle" cross sections. The latter implies that x-ray measurements are made using well-collimated systems. For loosely collimated systems, the Beer-Lambert law of Equation $1 \mathrm{~b}$ begins to break down due to $\mathrm{x}$-ray scatter in the radiographs.

A CT image is a spatial map of $\mu$. Because the absorption of $x$ rays is energy dependent, the spectrum evolves as the radiation penetrates a sample. Typically, the low-energy $\mathrm{x}$-rays are preferentially absorbed, the average spectral energy increases, and thus $\mu$ decreases with depth, an effect referred to as "beam hardening." 
The dependence of $\mu$ on spectrum means that it is also dependent on the system used to measure it. For an EDS to identify a material, its algorithms need to know what the LACs of that material will be on that specific system. It would be impractical to evaluate every HME of interest on every model of every EDS, so it is necessary to measure the x-ray properties of the sample on one "material-characterization" system, and to use that measurement to predict how that sample would appear on other "target" EDS systems. On the EBS program, the Micro-CT systems at the Transportation Security Lab (TSL), Tyndall Air Force Base and LLNL have been used as the material-characterization systems.

The Micro-CT systems are dual-energy systems. That is, each system measures LACs using two spectra: a high-energy spectrum and a low-energy spectrum. The LACs for both spectra are directly proportional to the electron-density of the sample and also depend in a complex way on the atomic composition of the sample and on the shape of the spectrum used in the measurement (Equation 1b). The LACs from the low-energy spectrum (we call $\mu_{\mathrm{lo}}$ ) depend more on atomic composition than do the LACs from the high-energy spectrum $\left(\mu_{\mathrm{hi}}\right)$. Thus, variations in the ratio of low-energy LACs to high-energy LACs $\left(\mu_{\mathrm{lo}} / \mu_{\mathrm{hi}}\right)$ provide a measure of the atomic composition of the specimen.

Some of the EDSs are also dual-energy systems. However, while the low- and high-energy spectra for the Micro-CT systems are identical, or nearly so, the EDS spectra are neither the same as the Micro-CT spectra nor the same as those of other EDS systems. To use measurements taken on one characterization system to predict measurements on a second, non-identical, target system, it is necessary to develop a transfer function, or map, between measurements on the two systems.

An approach used on the EBS program for mapping the response of a dual-energy characterization system to a dual-energy EDS system has been to use the parameter, $\mathrm{Z}_{\mathrm{eff}}$ (defined below) to characterize the atomic composition of materials. This report specifically discusses two different effective-atomic-number indices, $\mathrm{Z}_{\mathrm{eff}}$ and $\mathrm{Z}_{\mathrm{e}}$, and uses a detailed notation to differentiate between the number as calculated from the composition of a material and a number that has been measured using a CT system. To limit confusion, we will begin by defining notation along with a number of other symbols that are used throughout.

\subsection{Definition of Symbols used in this Document}

For a single element, its atomic number, Z, is unambiguous. For compounds and mixtures we can approximate the x-ray behavior of the material by an "effective atomic number." Because we are discussing different measures of "effective Z," it is important to make the definition of the terms used in this document clear:

Effective Z: A number that uses a representative atomic number to approximate the energydependent absorption of a complex material. By "complex materials," we refer to any combination of molecular compounds or mixtures that consists of more than one element. We use the term "effective Z" to refer generically to implementations of this concept. The EBS program has used $Z_{\text {eff }}$. In this document we propose an alternative, $Z_{e}$; the various EDS vendors have their own methods.

$\mathbf{Z}_{\text {eff: }}$ is an effective $\mathrm{Z}$ that is currently used to describe known compounds using a traditional formula given in Equation 2 below, where the $\mathrm{a}_{\mathrm{i}}$ are the relative electron fractions contributed by the constituent atoms. That formula is somewhat arbitrary in that no such 
formula can be accurate over all materials and spectra. To date, on this program, we have used $\mathrm{Z}_{\mathrm{eff}}$ to approximate the effective number of the reference materials used in the calibration, where the parameter $p$ is assigned the value 3.8. In this choice for $p$, we have not optimized the Equation 2 formula for either the spectra or materials of concern.

$$
Z_{\text {eff }}=\sqrt[p]{\sum_{i} a_{i} Z_{i}^{p}}
$$

Equation 2

$\mathbf{L}_{\mathbf{Z}} \mathbf{e f f}:$ describes the atomic number of an unknown material as measured on the Micro-CT systems. ${ }^{\mathrm{L}} \mathrm{Z}_{\mathrm{eff}}$ is determined by comparing the spectral responses of the unknown material to those of the reference materials. ${ }^{L} Z_{\text {eff }}$ is interpolated from the $Z_{\text {eff }}$ numbers that have been assigned to the references. ${ }^{\mathrm{L}} \mathrm{Z}_{\mathrm{eff}}$ is a system-dependent parameter. Because the Micro-CT systems utilize nearly identical spectral responses, the ${ }^{\mathrm{L}} \mathrm{Z}_{\mathrm{eff}}$ from the Micro-CT systems are essentially the same. The response of a substantially different system can similarly be interpolated using LAC measurements from its reference standards. A system-specific mapping function then can be found to map values from the Micro-CT system to any other CT system so that sample measurements made on the Micro-CT system can predict measurements on that other system.

$\mathbf{Z}_{\mathrm{e}}$ : is proposed as a more accurate parameter, and as a physically-defined x-ray model of known compounds (e.g., the reference materials), based on the calculated x-ray properties of those compounds. The $\mathrm{Z}_{\mathrm{e}}$ model is designed to closely match the actual $\mathrm{x}$-ray absorption of each material over the spectral region of interest. Per se, it removes the arbitrariness and lack of optimization entailed in using $\mathrm{Z}_{\mathrm{eff}}$.

$\mathbf{L}_{\mathbf{Z}}$ : If the recommended "Ze" method of characterizing the material were adopted, the MicroCT determination of the X-ray properties of unknown materials, ${ }^{\mathrm{L}} \mathrm{Z}_{\mathrm{e}}$ could be calculated from the $Z_{\mathrm{e}}$ in exactly the same way ${ }^{\mathrm{L}} \mathrm{Z}_{\mathrm{eff}}$ has been interpolated from the $\mathrm{Z}_{\mathrm{eff}}$, except that the calibration scale of the reference materials will be based on $Z_{e}$ rather than $Z_{\text {eff. }}$ The notation would be changed from ${ }^{\mathrm{L}} \mathrm{Z}_{\text {eff }}$ to ${ }^{\mathrm{L}} \mathrm{Z}_{\mathrm{e}}$ to mark the transition to the updated method.

$\mu: \quad$ The linear attenuation coefficient in a material as defined in Equation 1a. In the EBS program, the units of $\mu$ are $\mathrm{mm}^{-1}$.

$\mu_{10}$ : The linear attenuation coefficient in a material for the low-energy spectrum of a dualenergy system.

$\mu_{\text {hi }}: \quad$ The linear attenuation coefficient in a material for the high-energy spectrum of a dualenergy system.

Ex: X-ray energy. In this document the x-ray energy is expressed in keV.

$\{\boldsymbol{\sigma}$ and $\rho\}$ : In general, the linear $\mathrm{x}$-ray absorption due to a thickness of any material is the product of a cross-sectional area $\sigma\left(\mathrm{cm}^{2} / \mathrm{unit}\right)$ and the density of the material $\rho$ in the beam (units $/ \mathrm{cm}^{3}$ ). "Units" can be electrons, atoms, or molecules, etc. In this paper we use moles of electrons as our "unit;" this allows us to work with "laboratory-sized" quantities of material. The cross-sections, $\sigma$, are strongly dependent on the atomic composition and on the x-ray energy. The densities, $\rho$, depend only on the material density.

$\mathbf{\rho}_{\mathbf{e}}$ : $\quad$ Electron density per unit volume. In this document $\rho_{\mathrm{e}}$ is expressed in moles of electrons per $\mathrm{cm}^{3} \cdot \rho_{\mathrm{e}}$ for an element can be simply calculated from the mass density $\rho_{\mathrm{m}}\left(\mathrm{g} / \mathrm{cm}^{3}\right)$ by 
$\rho_{\mathrm{e}}=\rho_{\mathrm{m}} *(\mathrm{Z} / \mathrm{A})$, where $\mathrm{Z}$ and $\mathrm{A}$ are the atomic number and atomic weight. For a complex material the electron density is just the sum of contributions from the constituent elements:

$$
\rho_{e}=\rho_{m} \sum_{i} w_{i} Z_{i} / A_{i}
$$

Equation 3

where $\mathrm{w}_{\mathrm{i}}$ is the numerical fraction of element $\mathrm{i}$ present in the compound, and $\mathrm{Z}_{\mathrm{i}}$ and $\mathrm{A}_{\mathrm{i}}$ are the atomic number and atomic weight of that atom.

$\boldsymbol{\sigma}_{\mathrm{e}}: \quad$ The X-ray cross-section for a specified atomic number, $\mathrm{Z}$, and a given number of electrons. In this document, $\sigma_{\mathrm{e}}$ is expressed in $\mathrm{cm}^{2}$ per mole of electrons. $\sigma_{\mathrm{e}}$ depends strongly on the atomic number of a material, Z, and on the x-ray energy, Ex. For clarity, this may be shown expressly as $\sigma_{\mathrm{e}}(\mathrm{Z}, \mathrm{Ex})$. In this document $\sigma_{\mathrm{e}}$ is interpolated from the Livermore Evaluated Photon Data Library [1] with the conversion of the unit of charge from a single electron to a mole of electrons. For a complex material:

$$
\sigma_{e}=\sum_{i} b_{i} \sigma\left(Z_{i}\right)
$$

Equation 4

where $\sigma\left(Z_{i}\right)$ is the published cross section of the element, $Z_{i}$, and $b_{i}$ is the fraction of electrons contributed by the element $\mathrm{Z}_{\mathrm{i}}$.

$\boldsymbol{\sigma}_{\mathrm{Ze}}$ : The X-ray cross-section ( $\mathrm{cm}^{2}$ per mole of electrons) for a compound or mixture with an effective atomic number $Z_{\mathrm{e}}$. For clarity, this may be shown expressly as $\sigma_{\mathrm{Ze}}(\mathrm{Ze}, \mathrm{Ex})$.

\subsection{How $Z_{\text {eff }}$ has been used on this program to characterize HMEs}

The current program strategy to predict the EDS measurements on an HME based on Micro-CT measurements can be summarized as follows (the details of steps 4-6 are elaborated in Section 2.2):

\section{Calibrate the characterization and target systems:}

1. Establish a set of reference materials whose effective Z's and densities span the compositional range of materials of interest.

2. Assign each reference material an "effective atomic number" parameter, $Z_{\text {eff }}$, to represent its material composition.

3. Measure the dual-energy LACs, $\mu_{\mathrm{lo}}$ and $\mu_{\mathrm{hi}}$, for each reference material on the characterization system and on each target system.

4. For each system, approximate the relationship between the $Z_{\text {eff }}$ of the references and the measured LAC ratio $\left(\mu_{\mathrm{lo}} / \mu_{\mathrm{hi}}\right)$ by an interpolating polynomial.

\section{Measure the $\mu_{\mathrm{hi}}$ and ${ }^{\mathrm{L}} \mathrm{Z}_{\text {eff }}$ of the HME:}

5. Measure the LACs of the HME on the characterization system.

6. Use the Micro-CT polynomial function from step 4 to interpolate ${ }^{\mathrm{L}} \mathrm{Z}_{\text {eff }}$ of the HME from the $\mu_{\mathrm{lo}} / \mu_{\mathrm{hi}}$ ratio.

Calculate the EDS $\mu_{\mathrm{lo}}$ and $\mu_{\mathrm{hi}}$ of the HME from the ${ }^{\mathrm{L}} \mathrm{Z}_{\mathrm{eff}}$ and $\mu_{\mathrm{hi}}$ of the Micro-CT: 
7. Use the EDS polynomial function from step 4 to interpolate the $\operatorname{EDS} \mu_{\mathrm{lo}} / \mu_{\mathrm{hi}}$ ratio from the measured $\mathrm{Z}_{\text {eff. }}$.

8. Obtain the $\mu_{\mathrm{hi}}$ of the EDS from that of the Micro-CT (for improved accuracy, this relationship can be calibrated).

9. Calculate the $\mu_{\mathrm{lo}}$ of the EDS from its $\mu_{\mathrm{hi}}$ and the ratio from step 7 .

In this paper, we are specifically concerned with the techniques used to implement Step 2. As we will show, $Z_{\text {eff }}$ is defined in a way that makes it an incomplete, ambiguous, and imprecise representation of the $\mathrm{x}$-ray properties of complex materials. Because $\mathrm{Z}_{\mathrm{eff}}$ lies at the core of the response-mapping strategy, these deficiencies affect the accuracy with which measurements on the Micro-CT system can predict measurements on the EDS.

\subsection{Measurement of ${ }^{L} Z_{\text {eff }}$ based on the LACs and $Z_{\text {eff }}$ 's of the reference samples}

The experimental configuration of the Micro-CT systems provides two physically separate layers for the measurements [4]. The HME specimen is contained in a bottle on the top layer of a rotating sample holder (the "carousel"). The (nominally) 1/2-inch diameter reference samples are on the lower layer. A number of reference samples were chosen for use on this program [5]. For the measurements described here, each layer is imaged using an X-ray fan collimated through a narrow slit. The selected materials, and their assigned $Z_{\text {eff }}$ 's, are shown in Table 1.

Table 1. A list of the reference materials used on the Micro-CT and their assigned $Z_{\text {eff }}$ parameters. The ethanol and water are in polypropylene bottles.

\begin{tabular}{|c|c|l|}
\hline Material & $\mathbf{Z}_{\text {eff }}$ & \multicolumn{1}{|c|}{ Nominal Formulation } \\
\hline Graphite & 6.00 & $\mathrm{C}$ \\
\hline Ethanol & 6.53 & $95 \%$ Denatured Ethanol \\
\hline Delrin & 7.07 & $\mathrm{H}_{2} \mathrm{CO}$ \\
\hline Water & 7.54 & $\mathrm{H}_{2} \mathrm{O}$ \\
\hline Teflon & 8.50 & $\mathrm{CF}_{2}$ \\
\hline Aluminum & 13.00 & 6061 aluminum alloy \\
\hline
\end{tabular}

The interpolation of the measured ${ }^{\mathrm{L}} \mathrm{Z}_{\text {eff }}$ number for a hypothetical HME is illustrated in Figure 1. The measurements from the reference samples are shown as blue and red data points. Their measured LAC ratios $\left(\mu_{\mathrm{lo}} / \mu_{\mathrm{hi}}\right)$ and assigned $\mathrm{Z}_{\mathrm{eff}}$ 's are given on the horizontal and vertical axes respectively. The interpolation function is implemented as two separate polynomials (one each for the low- and high-Zeff ends of the data); the Delrin measurement is common to both fits [6].

For this graph, actual Micro-CT data have been used for the reference measurements. However, the HME measurement is hypothetical. We have assumed that the measured $\mu_{\mathrm{lo}} / \mu_{\mathrm{hi}}$ ratio for the hypothetical sample is 1.8 . The ${ }^{\mathrm{L}} \mathrm{Z}_{\mathrm{eff}}$ for this specimen is then interpolated from the upper (red) curve as 9.74 . The notation ${ }^{\mathrm{L}} \mathrm{Z}_{\text {eff }}$ indicates that this is an empirically measured number that has been interpolated from a measurement scale based on the calculated parameter $Z_{\text {eff. }}$. 


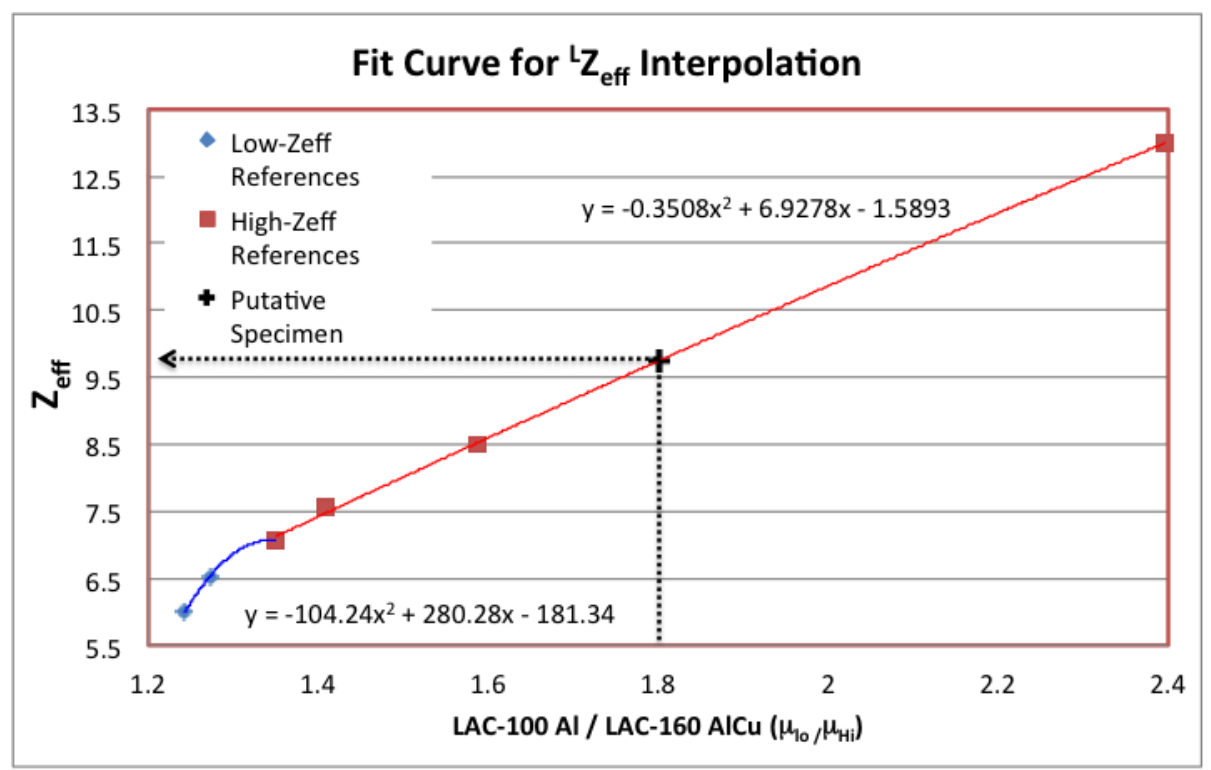

Figure 1. The method for measuring the ${ }^{\mathrm{L}} \mathrm{Z}_{\text {eff }}$ of an HME (steps $4-6$ in the text) is illustrated in this graph. The interpolation formulas for the low- and high- ends of the data are shown explicitly. The reference measurements are taken from LLNL Micro-CT data. The measurements on the "HME sample" are hypothetical.

\subsection{Discussion of the $\mathrm{Z}_{\mathrm{eff}}$ Parameter}

One of the key requirements for x-ray detection of an HME is to provide a representation of the chemical composition of the material as it appears in X-ray measurements. Every element (referred to by its atomic number, $\mathrm{Z}$ ) has a unique signature in the way it absorbs X-ray radiation. For example, high-Z elements have greater absorption, particularly at the lower x-ray energies. A compound or mixture of materials exhibits absorption that is a blend of the absorption from its constituent elements and will, in its energy dependence, resemble elements somewhere between the highest and lowest Z's present in the mixture. Because of the complexity of components involved in x-ray absorption, it is not possible to exactly represent any compound material as a single element, or using any single parameter. However, it can be possible, and useful, to approximate the absorption by a single parameter provided that parameter is associated with a model that specifies the energy-dependent absorption of that material, and that provides a means to optimize and evaluate the accuracy of the model.

The technique that has been used to date on this program has its historical roots in medical x-ray technology. In 1937, to estimate (without doing detailed and computationally-intensive calculations) where a particular material's absorption properties would fall on an "effective-Z" scale, W. V. Mayneord [7] proposed the Equation 2 expression with a power parameter of $\mathrm{p}=2.94$ for approximating the absorption of carbohydrates.

$$
Z_{\text {eff }}={ }^{p} \overline{{ }_{i} a_{i} Z_{i}^{p}}
$$

Equation 2

The $\mathrm{a}_{\mathrm{i}}$ are the relative electron fractions contributed by the constituent atoms. Since that time, this formula has been used as a convenient "rule-of-thumb," though the exact choice of the 
exponent, $\mathrm{p}$, has varied from observer to observer. Indeed, for best results, $\mathrm{p}$ should be chosen to fit the desired range of materials and spectra [8-13]. Given an optimal selection of $p$, this method has proven useful in the medical field for interpolating properties on specific systems and within the relatively narrow, and low-Z, range of materials of biological interest. If higher-Z components are present in the materials, it becomes very difficult to fit Equation 2 to materials of interest. For example, McCullough [10] warns that:

... even for the specific sub-components of the cross-sections, no single formula of the... $<$ Equation-2 >> ... form can describe the variations of those sub-components ...to the accuracy desired by any analysis relevant to CT scanning.

As discussed above, the strategy for the EBS has been to analytically interpolate an effective ${ }^{\mathrm{L}} \mathrm{Z}_{\mathrm{eff}}$ for an HME based on LAC ratios measured on reference materials, notably graphite, ethanol, Delrin, water, Teflon, and aluminum. The independent variable $\left(Z_{\text {eff }}\right)$ for the references was assigned using the $Z_{\text {eff }}$ formula (Equation 2 ) with the power $p=3.8$, as suggested by TSL [5]. The interpolated effective- $Z$ for the HME is called ${ }^{\mathrm{L}} \mathrm{Z}_{\text {eff. }}$ Unless otherwise specified, all $\mathrm{Z}_{\text {eff }}$ values in this document are calculated using $\mathrm{p}=3.8$.

The use of the $Z_{\text {eff }}$ equation to provide a characterization of the $\mathrm{x}$-ray properties for complex materials raises several issues.

\section{The use of the $Z_{\text {eff }}$ equation is inherently problematic}

The $\mathrm{Z}_{\text {eff }}$ equation was introduced as a computational tool to estimate the $\mathrm{x}$-ray absorption properties of complex materials at a time when detailed computation of the energy-dependent coefficients would have been difficult and expensive. It has likely not evolved, because its primary constituency, medical physics, has not needed to provide an accurate scale for as broad a range of materials as addressed by this program. Issues with the use of this formula include:

1. Any selection of the exponent " $p$ " in Equation 2 will not fit all compounds equally well. The selection of " $p$ " is a compromise. If the spectral and compositional range is limited, a "best value" can be chosen. Evaluating the accuracy of that choice is problematic because there is no specified relationship between $Z_{\text {eff }}$ and the $x$-ray properties (see point 4 below). As new HME's become of interest, and particularly if more high-Z components are used in the HME samples, the choice of "p" may need to be revisited. That would be disruptive to the continuity of the measurement strategy.

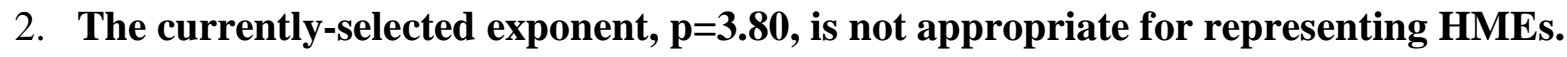
As shown in McCullough [10] an exponent of 3.8 is only representative of the Zdependence of the photoelectric absorption, and will consistently over-estimate the $Z_{\text {eff }}$ for other materials with high-Z constituents. Typical HME are dominated more by Compton than photoelectric absorption, and an optimal compromise would use a smaller exponent.

3. The calculated $Z_{\text {eff }}$ is an inherently ambiguous indicator of the $x$-ray absorption properties of materials. The simple way to see this is to look at Equation 2. An almost unlimited number of constituents could be compositionally adjusted to fit a specific $\mathrm{Z}_{\mathrm{eff}}$ number as long as that number is somewhere between the lowest and highest $\mathrm{Z}$ of the constituents selected. These varied compounds will, however, each have different X-ray absorption properties. 


\section{There is no defined way to get from the calculated " $Z_{\text {eff" }}$ " to the $x$-ray absorption}

properties of the material. We need the "effective-Z" to describe physical propertiesthe energy-dependent x-ray absorption - of an HME. Without a definition of the x-ray absorption that is specified by the $\mathrm{Z}_{\mathrm{eff}}$, we can say neither what those properties are, nor whether the selected number accurately describes those properties. Put simply, we do not have a physical model for $\mathrm{Z}_{\mathrm{eff}}$.

There is a considerable overlap between points 3 and 4, and it is worth illustrating the issue. For example, few would dispute that a material with an effective $Z$ of 10 ought to appear the same as a compact sample of the element neon $(Z=10)$. For example, if a small amount of iron $(Z=26)$ is added to a carbon substrate $(Z=6)$, to raise the $Z_{\text {eff }}$ of that sample to 10.0 , the absorption cross sections of that material ought to closely track the cross sections of neon.

If iron is added to a carbon base, with a weight fraction of $2.44 \%$, the $\mathrm{Z}_{\mathrm{eff}}$ calculated by Equation 2 (using $\mathrm{p}=3.8$ ) is 10. In Figure 2, the total $\mathrm{x}$-ray cross sections of neon (red line) and the putative $Z_{\text {eff }}=10$ compound (blue line) are compared; clearly, the low-energy absorption is much lower in the putative composite than in the neon (the black dotted line will be discussed later).

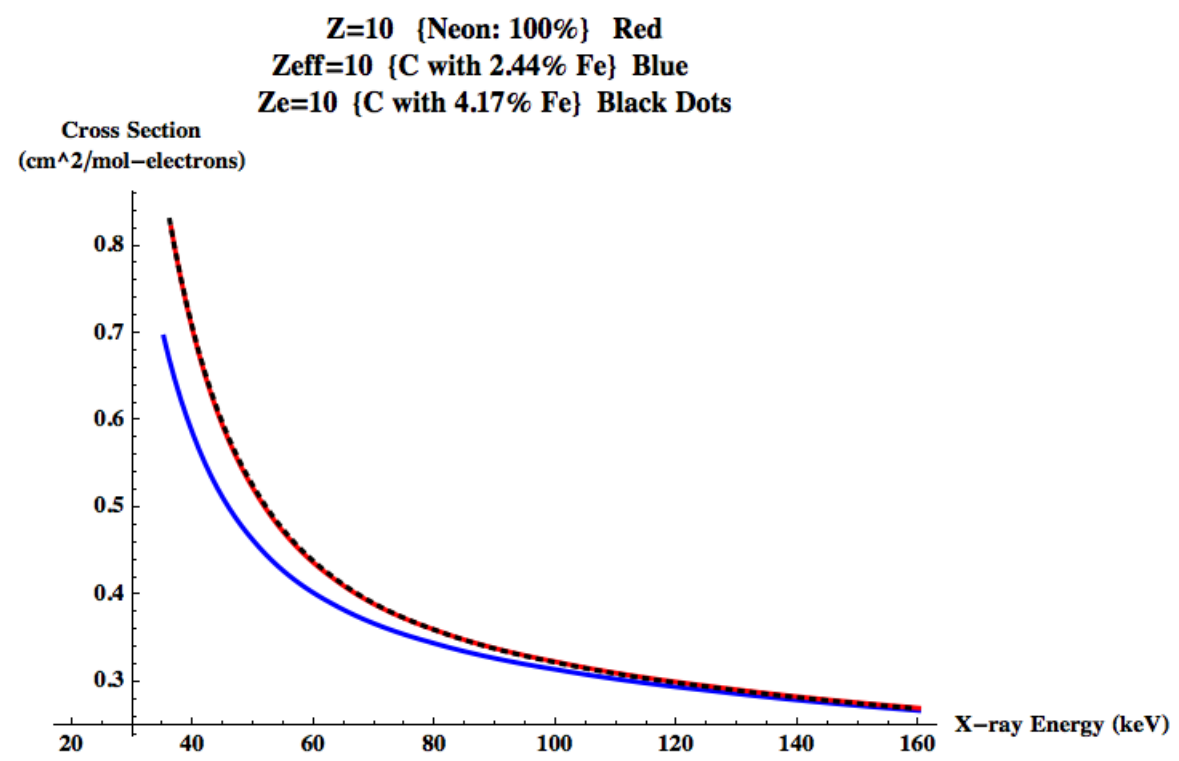

Figure 2. The $x$-ray cross-sections are compared for Neon $(Z=10)$ and for a carbon composite having $2.44 \%$ iron by weight $\left(Z_{\text {eff }}=10\right.$. $)$. At the low-energy end of the graph, the cross section for the composite is about $30 \%$ lower than that of the Neon. Clearly these material responses are significantly different. The cross section for $Z_{e}=10$, calculated using the proposed alternative technique (black dots), closely follows that of neon.

The contrast between the elements used in this mix $(Z=6$ and 26) is large, but it illustrates the point: $\mathrm{Z}_{\mathrm{eff}}$ is ambiguous. Any given $\mathrm{Z}_{\mathrm{eff}}$ number can represent a range of materials and a range of $\mathrm{x}$-ray properties. This means that $\mathrm{Z}_{\mathrm{eff}}$ cannot precisely specify the $\mathrm{x}$-ray properties of the references, and therefore ${ }^{\mathrm{L}} \mathrm{Z}_{\text {eff }}$, the measurement of an HME that is interpolated from the $\mathrm{Z}_{\text {eff }}$, cannot accurately convey the properties of that material to an EDS vendor.

An exponent " $p$ " in Equation 2 could be found to make this particular choice of ingredients match, however another selection of materials would then present a poor match. The fundamental point is that Equation 2 is not a physical model for a material's response - it 
provides no formula for calculating a unique $\mathrm{x}$-ray response for a specified $\mathrm{Z}_{\mathrm{eff}}$, and no means to verify that response.

In the next section, we present an alternative method for associating an effective atomic number, $Z_{e}$, with a specific material. Using that approach, which is based on a physical model, we find that a $Z_{e}$ of 10.0 corresponds to the addition of $4.17 \%$ iron by weight to the carbon. In Figure 2, it is clear that the cross-section for a $\mathrm{C} / \mathrm{Fe}$ material with $\mathrm{Z}_{\mathrm{e}}=10$ (black dots) is almost indistinguishable from that of neon.

\subsection{An Alternative Approach to Selecting the "Effective Z"}

There is a simple alternative, $\mathrm{Z}_{\mathrm{e}}$, that eliminates the problems in the previous section resulting from the $\mathrm{Z}_{\mathrm{eff}}$ formulation. There are two steps:

- Provide a physically meaningful definition for the effective $\mathrm{Z}, \mathrm{Z}_{\mathrm{e}}$, and for the corresponding x-ray cross-section: $\sigma_{\mathrm{Ze}}(\mathrm{Ze}, \mathrm{Ex})$.

- Provide an algorithm for determining $\mathrm{Z}_{\mathrm{e}}$ for an arbitrary known compound or mixture of elements.

\subsection{Define $Z_{e}$ and $\sigma_{Z e}(Z e, E x)$}

In x-ray physics, a material is described by its energy-dependent cross-section and electron density. Therefore, to have any physical meaning, $Z_{\mathrm{e}}$ must be defined by specifying $\sigma_{\mathrm{Ze}}\left(Z_{\mathrm{e}}\right.$, Ex). Our definition of $\sigma_{\mathrm{Ze}}\left(Z_{\mathrm{e}}, \mathrm{Ex}\right)$ is extremely simple: it is a linear interpolation between $\sigma_{\mathrm{e}}(\mathrm{Z}, \mathrm{Ex})$ and $\sigma_{\mathrm{e}}(\mathrm{Z}+1, \mathrm{Ex})$ where $\sigma_{\mathrm{e}}(\mathrm{Z}, \mathrm{Ex})$ is interpolated directly from any standard set of $\mathrm{x}$-ray absorption tables [e.g., 1-3]. To be specific:

a. For integer $\mathrm{Z}_{\mathrm{e}}$, the energy dependent $\mathrm{x}$-ray cross-section of a material is exactly that of the corresponding element with atomic number $\mathrm{Z}$, i.e.:

Integer Ze:

$$
\sigma_{Z e}(Z e, E x)=\sigma_{e}(Z e, E x)
$$

Equation $5 a$

b. For non-integer $Z_{\mathrm{e}}$, the cross-section, $\sigma_{\mathrm{Ze}}$ is obtained from a proportionate mixture of the cross-sections from the immediately adjacent elements. For example, a $\mathrm{Z}$ of 8.3 would be a 70/30 blend of Oxygen $(Z=8)$ and Fluorine $(Z=9)$. The $x$-ray cross-sections for this material can then be calculated:

$$
\sigma_{Z e}(8.3, E x)=0.7 \sigma_{e}(O, E x)+0.3 \sigma_{e}(F, E x)
$$

This definition can be written more formally. If we express the $Z_{e}$ as a real number with an integer part (Z) and a fractional part (frac), then,

Non-integer Ze:

$$
\sigma_{Z e}(Z . f r a c, E x)=(1-f r a c) * \sigma_{e}(Z, E x)+f r a c * \sigma_{e}(Z+1, E x)
$$

Equation $5 b$ 
In practice, only Equation $5 \mathrm{~b}$ is required, because, if $\mathrm{Z}_{\mathrm{e}}$ is an integer, Equation $5 \mathrm{~b}$ reduces to Equation 5a. Note that "frac" refers to the fraction of electrons, rather than the fraction of atoms, contributed to the material from the $\mathrm{Z}+1$ element.

Equation $5 \mathrm{~b}$ defines $\mathrm{Z}_{\mathrm{e}}:$ : A material with an effective atomic number, $Z_{e}$, has the energydependent cross-section of Equation $5 b$.

Note that this definition of $\mathrm{Z}_{\mathrm{e}}$ is not an approximation. It is a specific definition in terms of physical parameters. It is valid over the same range of parameters as the $\mathrm{x}$-ray absorption tables from which it is interpolated. Our knowledge of the $\mathrm{x}$-ray cross-section of $Z_{e}$, is neither more nor less accurate than those tables, which express the current knowledge of $\mathrm{x}$-ray interaction probabilities.

Combining Equations $1 \mathrm{~b}, 4$, and $5 \mathrm{~b}$ for compounds, the familiar exponential attenuation of $\mathrm{x}$-ray intensity with thickness, $\mathrm{x}$, can be written:

$$
I E x=I o \operatorname{Ex} \operatorname{Exp}\left[-\sigma_{Z e}\left(Z_{e}, E x\right)\right] \quad \rho_{e} x d x
$$

Equation 6

Two obvious questions arise:

1. How do you find an optimal $\mathrm{Z}_{\mathrm{e}}$ for a specified compound or mixture?

2. Under what conditions can the x-ray properties of any specific material be accurately approximated by $\mathrm{Z}_{\mathrm{e}}$ (or any other effective- $\mathrm{Z}$ )?

\subsection{Calculating $Z_{e}$ for an Arbitrary Compound or Mixture}

For compounds and mixtures of elements, $\sigma(\mathrm{Ex})$ is just the proportionate sum of the cross sections of the constituent atoms. Elaborating on Equation 4, for a material with $\mathrm{N}$ types of atoms, with atom $\mathrm{Z}_{\mathrm{i}}$ present in the proportion $\mathrm{n}_{\mathrm{i}}$ :

$$
\begin{gathered}
\sigma(\text { material }, E x)=\sum_{i}^{N} b_{i} \sigma\left(Z_{i}, E x\right) \\
b_{i}=n_{i} Z_{i} / \sum_{i=1}^{N} n_{i} Z_{i}
\end{gathered}
$$

Equation 7

As an example, for water, $\mathrm{H}_{2} \mathrm{O}, \mathrm{N}=2, \mathrm{Z}_{1}=1, \mathrm{Z}_{2}=8, \mathrm{n}_{1}=2, \mathrm{n}_{2}=1$, so

$$
\sigma(\text { water }, E x)=\frac{2}{10} \sigma(H, E x)+\frac{8}{10} \sigma(O, E x)
$$

The procedure for finding $Z_{e}$ is then conceptually simple - the best $Z_{e}$ provides the best agreement between $\sigma_{\mathrm{Ze}}\left(\mathrm{Z}_{\mathrm{e}}, \mathrm{Ex}\right)$ and $\sigma$ (material, Ex). Another way to state the principle is to "use the $\mathrm{Z}_{\mathrm{e}}$ that will provide the best match for the (Equation 7) calculated cross-section of the known material."

A straightforward approach would be to use a $\mathrm{Z}_{\mathrm{e}}$ that has the best least-squares-fit to the crosssection over the $\mathrm{x}$-ray range of interest. However, we suggest using a finesse here that provides a more robust result. 
Recall that any effective-Z, including $Z_{\mathrm{e}}$, can only provide an approximation to the energydependent cross-section of a compound. For any compound, that approximation will eventually fall apart at the lowest energies largely because the ratio of the coherent and photoelectric crosssections differs between the actual compound, and that calculated via the $Z_{\mathrm{e}}$ definition. That discrepancy will occur at higher energies if the material is a mixture of very-high- $Z$ and verylow-Z elements. The combination of the high- $Z$ (iron) with the low-Z (carbon) in Figure 2 was deliberately chosen to emphasize that effect.

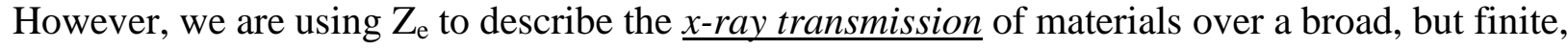
spectral region. The $\mathrm{x}$-ray transmission of an object rapidly decreases at the lowest energies. If there is significant $\mathrm{x}$-ray transmission at the lowest energies, we would like to find the best compromise that includes transmission at those low energies. But, where there is very little transmission at low-energies, we want to decrease the weighting given to the lowest energies.

The way we have handled this issue is to select $Z_{\mathrm{e}}$ to obtain the best least-squares-fit for the transmission of $\mathrm{x}$ rays through a material of moderate thickness. For the EBS program, $2.5 \mathrm{~g} / \mathrm{cm}^{2}$ of material (essentially 1-inch of water) seems appropriate, and has been used for the calculations in this paper. Note that the choice of thickness is not at all critical, however allowing the material absorption to weight the least-squares fit provides a self-adjusting cutoff for the lowest energies used in the calculation whenever higher- $Z$ constituents are present. This is illustrated in section 4.3. Use of transmission weighting also de-emphasizes the highest energies where there is less absorption and little Z-dependence in the absorption.

It is also important to apply the optimization over an appropriate spectral region. For EBS applications, the combination of source filtering and object attenuation transmits little radiation below $30 \mathrm{keV}$, and typical peak source energies are $160 \mathrm{kV}$, so we have done our optimization over a range of $30-160 \mathrm{keV}$.

The results of applying this algorithm to Teflon are illustrated in Figure 3, where a Ze of 8.43 is shown to closely fit the transmission of Teflon across the selected range. As shown earlier in Figure 2, the energy-dependent cross section for an Fe/C mixture, calculated using this algorithm, also fits the $Z_{\mathrm{e}}$ description very closely. 
Teflon: Red; Ze $=8.43$ : Black Pts.

$Z=8$ : Blue; $Z=9$ : Green

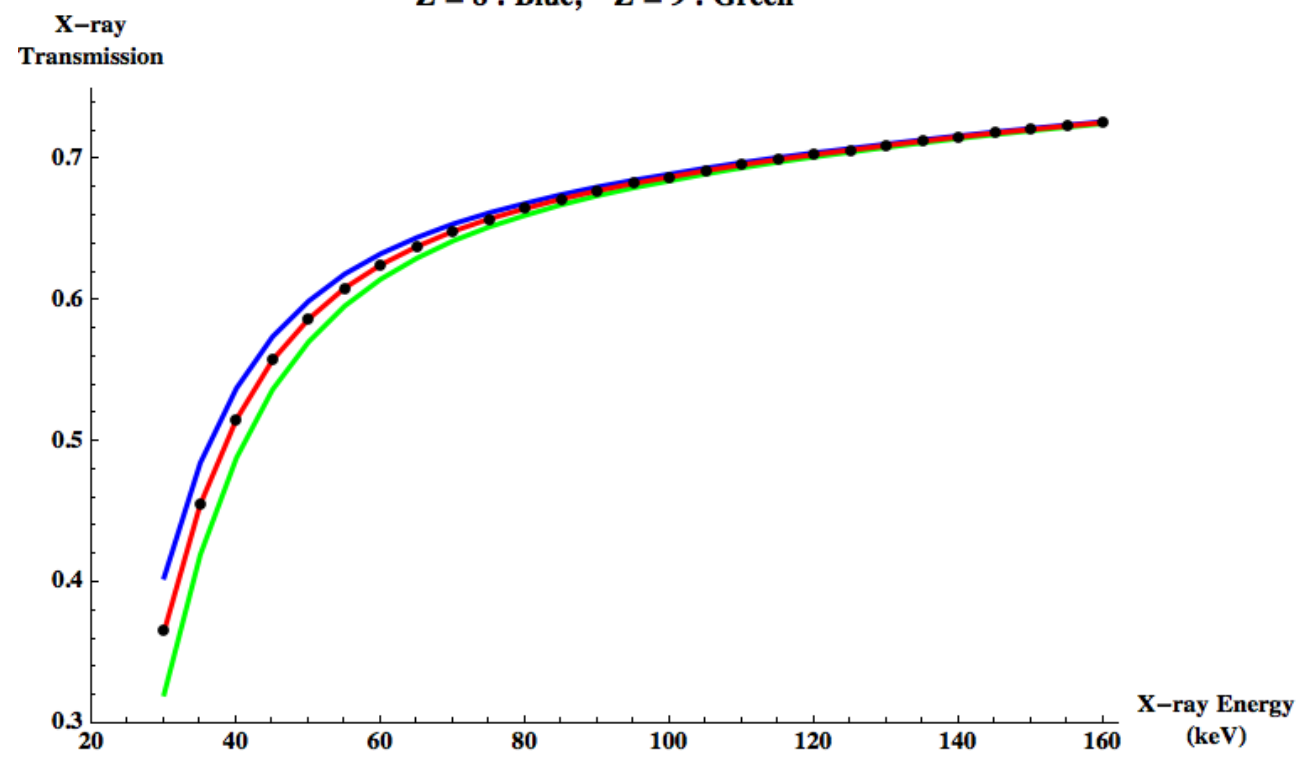

Figure 3. The x-ray transmission through a $(2.5 \mathrm{~g} / \mathrm{cm} 2)$ sheet of Teflon (red) is closely matched by the transmission calculated for Ze of 8.43 (black points). For comparison, transmissions through the same areal mass of Oxygen $(\mathrm{z}=8)$ and Fluorine $(\mathrm{z}=9)$ are shown.

\subsection{Range of Applicability of Effective-Z Models}

$Z_{e}$, or any effective- $Z$, is an approximate description of a material. For many materials, and spectral regions - and for most materials of interest to the EBS Program - it can be an excellent approximation of the energy-dependent $\mathrm{x}$-ray absorption. Two situations illustrated in Figure 4 may present problems.

\section{Cross Section Ratio: Effective-Z model to Actual Material Blue: Teflon}

Red: $4.2 \%$ Iron-Carbon Mixture Cross-Section Ratio

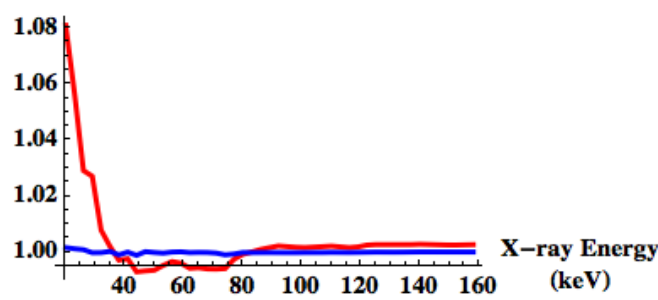

\section{$\mathrm{X}$-ray transmission through $2.5 \mathrm{~g} / \mathrm{cm}^{\wedge} 2$ sample Blue: Teflon}

Red: $4.2 \%$ Iron-Carbon Mixture

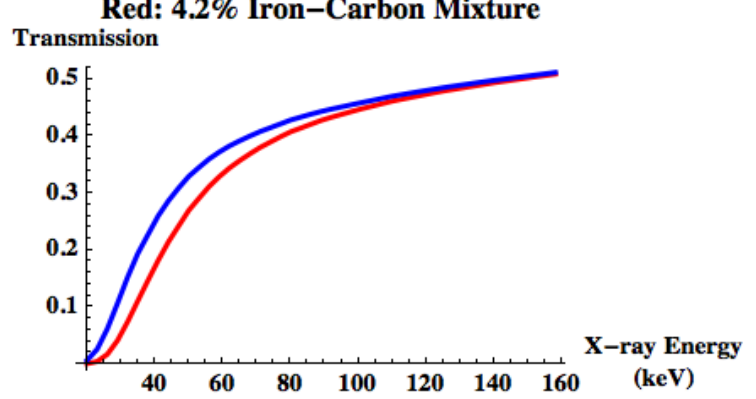

Figure 4. For Teflon, the effective-Z provides an excellent approximation to the cross-section over a broad range of energies. However, for the fictional Fe/C mixture analyzed in the text, $\mathrm{Z}=10$ is accurate down to about $35 \mathrm{keV}$. Below $30 \mathrm{keV}$, the cross-section ratio quickly diverges. Optimizing Ze according to the transmission through a material effectively eliminates sensitivity below $30 \mathrm{keV}$. 
First, for any complex material, the effective- $Z$ model will have a low-energy limit, below which the actual cross section and effective- $Z$ cross section diverge. The left panel of Figure 4 illustrates this issue for Teflon and for the iron/carbon mixture that was discussed above. The plot shows the ratio of the effective- $Z$ models, $\sigma_{\mathrm{e}}\left(Z_{\mathrm{e}}, \mathrm{Ex}\right)$, to the calculated cross section, $\sigma$ (material, Ex), for the actual materials. For Teflon, which is composed of the relatively low-Z materials carbon and fluorine, the cross sections match very closely down to $20 \mathrm{keV}$. For the posited iron/carbon mixture, the ratio diverges below $35 \mathrm{keV}$.

However, the algorithm used to optimize $\mathrm{Z}_{\mathrm{e}}$, calculates transmission differences through a 2.5 $\mathrm{g} / \mathrm{cm}^{2}$ sample. As shown in the right panel of Figure 4, the transmission through the iron/carbon mixture cuts off at about $30 \mathrm{keV}$, so the ratio divergence would have little effect on the calculated value of $Z_{\mathrm{e}}$. In the field, EDS scanners would only see a difference between the effective- $Z$ model and the material for thin sheets, unshielded by other material, and viewed perpendicular to the surface of the sheet. Thus, even for a fairly radical high-Z/low-Z mixture, the $Z_{\mathrm{e}}$ model remains robust. At some point, as the contrast between the constituent materials increases, the model will break and an effective- $Z$ model will not be useful.

The hypothetical iron/carbon sample is just at the threshold where an effective-Z model becomes inappropriate, and serves as an illustration that, if we use an effective- $Z$ model for the materials being characterized, we need to be vigilant as small amounts of high-Z elements are added to the compound.

The second problem that may arise is the inclusion of elements with absorption edges in the middle of the observing spectrum. The k-absorption edge of tin $(Z=50)$ is just below $30 \mathrm{keV}$ and should not present a problem. However elements significantly above that, such as lead or tungsten, could present a "jagged" transmission characteristic that would be very sensitive to the low-energy cutoff of the probing spectrum. Again, in that situation, an effective-Z model would not be useful. Like any tool, effective-Z models need to be used thoughtfully.

\subsection{Are the proposed changes Better and are they "Significant?"}

$\mathrm{Z}_{\mathrm{e}}$ is qualitatively "better" than $\mathrm{Z}_{\mathrm{eff}}$ in that the $\mathrm{Z}_{\mathrm{e}}$ calculation provides a model to define the $\mathrm{x}$-ray transmission properties of the material having that effective atomic number. $Z_{\text {eff }}$ is, in fact, mathematically ambiguous. $Z_{\mathrm{e}}$ provides a valid single-valued physical model for every material.

$\mathrm{Z}_{\mathrm{e}}$ is quantitatively better because it provides an accurate approximation to the $\mathrm{x}$-ray transmission for any specified material. Its accuracy is built in by using the best-fit algorithm in its implementation.

Evaluation of Micro-CT system performance has shown measurement consistency for $\mu_{\mathrm{lo}}$ and $\mu_{\mathrm{hi}}$ on the order of $0.6 \%$ and $0.1 \%$ respectively [14]. Additionally, evaluation of HMEs in this program has indicated the need to achieve measurement precision for $\mu_{\mathrm{hi}}$ on the order of $0.5 \%$ [15]. From the slope of the upper segment of the Figure-1 graph, we can see that $0.6 \%$ variation in the measurement of the LAC ratio, corresponds to a variation in the $\mathrm{Z}_{\mathrm{eff}}$ of about 0.04 .

Table 2 provides a perspective on the magnitude of the adjustments resulting from using the $Z_{\mathrm{e}}$ process for calculating the "effective-Z". The table assumes that the nominal molecular compositions are correct. If the program objective is to measure material properties, the differences exceed the figure-of-merit $(\Delta \mathrm{Z} \sim 0.04)$. This suggests that, at present, the accuracy 
and consistency of our "Zeff ruler" is not as precise as our LAC measurements and that a more accurate calibration would be appropriate.

Table 2. Comparisons of the $Z_{\text {eff }}$ that are used on the current program and the improved $Z_{e}$ values using the approach outlined above.

\begin{tabular}{|c|c|c|c|l|}
\hline Material & $\mathbf{Z}_{\text {eff }}$ & $\mathbf{Z e}$ & Difference & \multicolumn{1}{|c|}{ Formulation } \\
\hline Ethanol & 6.53 & 6.37 & -0.16 & $100 \%$ Ethanol \\
\hline Delrin & 7.07 & 7.01 & -0.06 & $\mathrm{H}_{2} \mathrm{CO}$ \\
\hline Water & 7.54 & 7.43 & -0.11 & $\mathrm{H}_{2} \mathrm{O}$ \\
\hline Teflon & 8.50 & 8.43 & -0.07 & $\mathrm{CF}_{2}$ \\
\hline
\end{tabular}

If the number ${ }^{\mathrm{L}} \mathrm{Z}_{\text {eff }}$ is intended to convey information about a specific sample, its ambiguity and lack of precision does not seem appropriate for its role. Note that the ambiguity becomes greater when higher- $Z$ elements are present in the sample.

The advantages of the proposed definition of $\mathrm{Z}_{\mathrm{e}}$ can be summarized as follows:

- Accuracy: The definition of $Z_{e}$ optimizes the effective- $Z$ for each material.

- Flexibility: If, and when, new HMEs are encountered, the selected algorithm does not need to be adjusted. The technique, by its definition, provides an optimized description for each material.

- Specificity: The effective atomic number, $Z_{\mathrm{e}}$, along with the electron density of a particular sample, $\rho_{\mathrm{e}}$, uniquely specifies the $\mathrm{x}$-ray absorption for each material.

\subsection{Summary and Recommendations}

- The $\mathrm{Z}_{\text {eff }}$ parameter does not accurately describe the $\mathrm{x}$-ray properties of materials. An alternative, $Z_{\mathrm{e}}$, provides a more accurate material description.

- To assure that the choice of an effective-atomic number parameter does not significantly impact the accuracy of data produced on this program, we recommend that the use of $Z_{\mathrm{eff}}$ be replaced, and that the more accurate $\mathrm{Z}_{\mathrm{e}}$ be used instead.

- At some point, the EBS program will need to move to a more precisely-defined set of reference materials to serve as the measurement standards for characterization of HME. That point would provide an opportunity to move the program to the use of the more accurate $Z_{\mathrm{e}}$ parameter (See Appendix). 


\subsection{References}

1) D.E. Cullen, S.T. Perkins, and J. A. Rathkopf, The 1989 Livermore Evaluated Photon Data Library (EPDL), UCRL-ID-103424, Lawrence Livermore National Laboratory (1990).

2) D.E. Cullen, J. H. Hubbell, and L. Kissel, EPDL97: The Evaluated Photon Data Library '97 Version, UCRL-ID-50400, Vol. 6, Rev. 5, Lawrence Livermore National Laboratory (1997).

3) J. H. Hubbell, and S. M. Seltzer, Tables of X-Ray Mass Attenuation Coefficients and Mass Energy-Absorption Coefficients from $1 \mathrm{keV}$ to $20 \mathrm{MeV}$ for Elements $Z=1$ to 92 and 48 Additional Substances of Dosimetric Interest, NISTIR 5632, 1996: http://www.nist.gov/pml/data/xraycoef/index.cfm

4) J. A. Smith, D. J. Schneberk, J. S. Kallman, H. E. Martz, Jr, D. Hoey, R. Krauss, R. Klueg, Documentation of the LLNL and Tyndall Micro-Computed-Tomography Systems (Micro-CT), LLNL-TR-421377, 2011.

5) R. Krauss, R. Klueg, Standard Operating Procedure: Industrial Computed Tomography System Data Collection of Home-Made Explosives, DHS/STD/TSL-XX-XX, 2009.

6) J. S. Kallman, D. J. Schneberk, H. E. Martz, Jr., Ratio of Two Energy Spectra Method and Reference Materials to determine Effective Z, LLNL-TR-XXXX (Draft), 2009.

7) M. V. Mayneord, the Significance of the Roentgen, Acta of the International Union Against Cancer, V ii, p. 271, 1937.

8) F. W. Spiers, Effective Atomic Number and Energy Absorption in Tissues, Br. J. Radiol., 19, 52, 1946.

9) J Weber, and K. J. van den Berge, The effective atomic number and the calculation of the composition of phantom materials, Br. J. Radiol., 42, 378-383, 1969.

10) E. C. McCullough, Photon attenuation in computed tomography, Medical Physics, Vol. 2. 6, Nov/Dec, 1975.

11) D. R. White, An Analysis of the Z-dependence of Photon and Electron Interactions, Phys. Med Biol., 1977, Vol. 22, 219-228, 1977.

12) N. C. Yang, P.K. Leichner, and W. G. Hawkins, Effective atomic numbers for low-energy total photon interactions in human tissues, Medical Physics, Vol. 14, No. 5, Sep/Oct 1987.

13) B. J. Heismann, J. Leppert, and K. Stierstorfer, Density and atomic number measurements with spectral $x$-ray attenuation method, Journal of Applied Physics, Vol. 94, No. 3, 2073-79, 2003.

14) J. S. Kallman, H.E.Martz and W.D. Brown, MicroCT Reference Materials QA Windows, LLNL-PRES-489954-DRAFT slide 7, July 2011.

15) H. E. Martz, Jr. C. R. Crawford, J. S. Kallman, Set 1 b Group 1 Information, LLNL-MI461695, 2011. 


\subsection{Appendix: How the Proposed Definition of Ze Impacts the Current HME Program}

- In conjunction with obtaining chemical assays of the "as deployed" reference materials, the effective- $Z$ would be recalculated using the $Z_{\mathrm{e}}$ definition. The magnitude of the impact on previous data should be assessed in view of those results.

- To bring previous work in line with the improved calibrations, the work based on the Zeff calibrations could be updated; however, it may be adequate to simply provide a "correction function" to adjust previous $Z_{\text {eff }}$ results to the more accurate $\mathrm{Ze}$.

- For the immediate future, the effective- $Z$ of the HME would be interpolated using the current interpolation technique, but with the assigned effective atomic number of the references based on the more accurate $Z_{\mathrm{e}}$ scale calculated using accurately assayed reference materials. These would be referred to as ${ }^{\mathrm{L}} \mathrm{Z}_{\mathrm{e}}$, where ${ }^{\mathrm{L}} \mathrm{Z}_{\mathrm{e}}$ is $\mathrm{Z}_{\mathrm{e}}$ measured using the current interpolation technique. For materials with known composition, $Z_{\mathrm{e}}$ can be calculated directly. That is, the x-ray cross section can be calculated based on published data [1-3].

\section{Effect of Changing the Effective-Z on Data Acquisition}

Micro-CT data acquisition is unchanged when we recalibrate the effective atomic number of our references.

\section{Effect of Changing Effective-Z on Image Reconstruction and Object Segmentation}

CT reconstruction and segmentation remain unchanged.

To effect the change in computing ${ }^{\mathrm{L}} Z_{\mathrm{e}}$ will require replacing the current $Z_{\text {eff }}$ values for the references in the characterization template spreadsheet with the $Z_{\mathrm{e}}$ values determined by the technique described above and based on the chemical assays of the as-deployed reference materials. 